\title{
RELAXAÇÕES CONVEXAS DE CONVERGÊNCIA GARANTIDA PARA O PROJETO DE CONTROLADORES PARA SISTEMAS NEBULOSOS DE TAKAGI-SUGENO
}

\author{
Vinícius F. Montagner* \\ montagne@smail.ufsm.br \\ Pedro L. D. Peres ${ }^{\dagger}$ \\ peres@dt.fee.unicamp.br \\ *Departamento de Processamento de Energia Elétrica \\ Universidade Federal de Santa Maria - UFSM \\ 97105-900, Santa Maria, RS, Brasil \\ †Departamento de Telemática \\ Faculdade de Engenharia Elétrica e de Computação \\ Universidade Estadual de Campinas - UNICAMP \\ CP 6101, 13081-970, Campinas, SP, Brasil
}

Ricardo C. L. F. Oliveira ${ }^{\dagger}$

ricfow@dt.fee.unicamp.br

\begin{abstract}
Convex Relaxations with Guaranteed Convergence for Control Design of Takagi-Sugeno Fuzzy Systems

This paper provides convex conditions with certificates of convergence for the design of state feedback controllers that quadratically stabilize and also ensure optimal $\mathscr{H}_{2}$ and $\mathscr{H}_{\infty}$ performances under quadratic stability for Takagi-Sugeno continuous-time fuzzy systems. The proposed conditions are formulated as parameterdependent linear matrix inequalities (LMIs) that have extra variables from Finsler's lemma and parameters belonging to the unit simplex. The fuzzy control law is written as a state feedback gain that is a homogeneous polynomial of degree $g$, encompassing the parallel distributed compensator as a special case when $g=1$. Algebraic properties of the system parameters and recent results of positive polynomials are used to construct LMI relaxations which, differently from most relaxations
\end{abstract}

\footnotetext{
Artigo submetido em 27/03/2009 (Id.: 00979)

Revisado em 29/05/2009

Aceito sob recomendação do Editor Associado Prof. Alexandre Bazanella
}

in the literature, asymptotically converge to a solution whenever such solution exists. Due to the degree of freedom obtained with the extra variables, the conditions presented in the paper are an improvement over earlier results based on Pólya's theorem and can be viewed as an alternative to the use of techniques based on the relaxation of quadratic forms. The numerical efficiency in terms of precision and computational effort is demonstrated by means of comparisons with other methods from the literature.

KEYWORDS: Takagi-Sugeno fuzzy systems, Linear matrix inequalities, Convergent relaxations, $\mathscr{H}_{2}$ control, $\mathscr{H}_{\infty}$ control.

\section{RESUMO}

Este artigo fornece condições convexas com convergência garantida para o projeto de controladores por realimentação de estados que estabilizam quadraticamente e também asseguram desempenhos ótimos $\mathscr{H}_{2}$ e $\mathscr{H}_{\infty}$ sob estabilidade quadrática para sistemas nebulosos de Takagi-Sugeno contínuos no tempo. As condições pro- 
postas são formuladas como desigualdades matriciais lineares (LMIs) dependentes de parâmetros com variáveis de folga provenientes do Lema de Finsler e com parâmetros pertencentes ao simplex unitário. A lei de controle nebulosa é dada por um ganho de realimentação de estados que é um polinômio homogêneo de grau $g$, que tem como caso particular o compensador paralelo distribuído quando $g=1$. Propriedades algébricas dos parâmetros do sistema e resultados recentes sobre positividade de polinômios são usados para construir relaxações LMIs que, diferentemente da maioria das relaxações da literatura, convergem assintoticamente para a solução sempre que esta existir. Graças ao grau de liberdade obtido pelo uso de variáveis de folga, as condições apresentadas no artigo representam avanços em relação a condições recentemente publicadas baseadas no Teorema de Pólya, e podem ser vistas como uma alternativa a técnicas baseadas na relaxação de formas quadráticas. A eficiência numérica em termos de precisão e esforço computacional é demonstrada por meio de comparações com outros métodos da literatura.

PALAVRAS-CHAVE: Sistemas nebulosos de TakagiSugeno, Desigualdades matriciais lineares, Relaxações convergentes, Controle $\mathscr{H}_{2}$, Controle $\mathscr{H}_{\infty}$.

\section{INTRODUÇÃO}

A procura por condições de projeto de controladores eficientes e gerais para sistemas não-lineares é um assunto de grande interesse. Nesse contexto, sistemas nebulosos de Takagi-Sugeno (T-S) têm um papel importante, pois permitem a representação de sistemas não-lineares por meio de combinação de modelos lineares, tornando-se uma ferramenta útil de modelagem, análise e controle de sistemas não-lineares (Wang et al., 1996; Tanaka et al., 1998a; Tanaka et al., 1998b; Teixeira e Zak, 1999; Johansson et al., 1999; Cao e Frank, 2000; Chen et al., 2000; Kim e Lee, 2000; Tanaka e Wang, 2001; Tuan et al., 2001; Lian et al., 2001; Liu e Zhang, 2003; Teixeira et al., 2003; Feng, 2006; Kumar et al., 2006).

A abordagem baseada em funções de Lyapunov é bastante utilizada para sistemas nebulosos de T-S, levando a condições de análise e síntese freqüentemente expressas na forma de desigualdades matriciais lineares (do inglês, Linear Matrix Inequalities - LMIs) (Boyd et al., 1994). Procedimentos de otimização baseados em LMIs podem ser resolvidos em tempo polinomial por algoritmos de convergência global (Sturm, 1999). Uma estrutura de controle bastante utilizada em sistemas nebulosos de T-S é o compensador paralelo distribuído, na qual um controlador é projetado para cada regra do modelo nebuloso, compondo um controlador global dado pela soma ponderada de controladores lineares. A estabilidade global do sistema é assegurada, por exemplo, pela existência de uma função de Lyapunov comum (Wang et al., 1996; Tanaka et al., 1998a; Tanaka e Wang, 2001). Essa estratégia de controle tem sido largamente utilizada, principalmente em realimentação de estados ou em realimentação dinâmica da saída. Graças à formulação LMI do problema, podem ser incorporadas restrições adicionais como alocação de pólos para cada subsistema linear, limitações da ação de controle, robustez a incertezas e atrasos (Boyd et al., 1994; Tanaka et al., 1998b; Cao e Frank, 2000; Chen et al., 2000; Tanaka e Wang, 2001; Nguang e Shi, 2003; Xu e Lam, 2005).

A existência de uma função de Lyapunov quadrática que assegura a estabilidade global do sistema nebuloso de TS em malha fechada utilizando um compensador paralelo distribuído é equivalente à existência de uma matriz simétrica definida positiva $W$ e de uma matriz linearmente dependente de parâmetros $Z(\alpha)$ resolvendo, para todo $\alpha$ (um vetor de parâmetros variantes no tempo pertencente ao simplex unitário), a LMI dependente de parâmetros que caracteriza a estabilizabilidade quadrática, dada por

$$
A(\alpha) W+W A(\alpha)^{\prime}+B(\alpha) Z(\alpha)+Z(\alpha)^{\prime} B(\alpha)^{\prime}<0
$$

em que $A(\alpha)$ e $B(\alpha)$ são expressas como combinações convexas das matrizes dos subsistemas do modelo nebuloso de T-S. Conforme discutido em Tuan et al. (2001), $Z(\alpha)$ - e, conseqüentemente, $K(\alpha)$ - podem ser restritas a matrizes linearmente dependentes de parâmetros, sem perda de generalidade. A solução dessa LMI dependente de parâmetros para todo $\alpha$ pertencente ao simplex unitário é, entretanto, um problema de dimensão infinita. Explorando o fato de esta LMI poder ser escrita como uma desigualdade matricial quadraticamente dependente de parâmetros, condições LMI de dimensão finita para resolver o problema podem ser obtidas (veja, por exemplo, Tanaka e Wang (2001, Seção 3.3)).

Condições suficientes que utilizam relaxações de formas quadráticas (i.e., variáveis de folga no lado direito das LMIs) podem ser encontradas em Kim e Lee (2000) e Liu e Zhang (2003). Outras condições suficientes na forma de LMIs para a estabilizabilidade quadrática têm sido propostas (Tanaka et al., 1998a; Teixeira et al., 2000; Tuan et al., 2001; Teixeira et al., 2003; Fang et al., 2006; Andrea et al., 2008), em geral reduzindo o conservadorismo em relação às condições anteriores, mas sem convergência garantida para a solução do problema da estabilizabilidade quadrática por meio de compensadores paralelos distribuídos. Somente recentemente o Teorema de Pólya foi aplicado à solução de (1) para 
produzir seqüências de LMIs que tendem assintoticamente a condições necessárias (Montagner, Oliveira e Peres, 2007; Sala e Ariño, 2007; Montagner et al., 2008). Em Sala e Ariño (2007), os autores usam variáveis de folga no lado direito das LMIs para melhorar as propriedades numéricas das relaxações do Teorema de Pólya. Uma comparação entre esses resultados no âmbito do compensador paralelo distribuído sob estabilidade quadrática pode ser vista em Montagner et al. (2009), que apresenta também condições de estabilizabilidade quadrática com custo garantido $\mathscr{H}_{\infty}$.

É interessante observar que, por um lado, vários resultados de LMIs aplicados a sistemas nebulosos de T-S são originários da teoria de controle robusto para sistemas variantes e invariantes no tempo, ao passo que diversas relaxações que asseguram a negatividade de uma LMI dependente de parâmetros apareceram primeiramente na literatura de sistemas nebulosos. Assim, por exemplo, o Teorema de Pólya (Hardy et al., 1952) aparece no contexto de LMIs dependentes de parâmetros primeiramente em Scherer (2003) (veja também Scherer (2005) e Scherer e Hol (2006)), sendo depois usado para construir relaxações LMIs assintoticamente necessárias para a existência de funções de Lyapunov afins assegurando a estabilidade robusta de sistemas lineares com incerteza politópica em Oliveira e Peres (2005). No contexto de relaxações para sistemas nebulosos T-S, os resultados apareceram depois (Montagner, Oliveira e Peres, 2007; Sala e Ariño, 2007; Montagner et al., 2008; Montagner et al., 2009). Já as relaxações baseadas em variáveis de folga do lado direito surgiram para sistemas nebulosos T-S, aplicadas à desigualdade (1) (Kim e Lee, 2000; Liu e Zhang, 2003), sendo depois aplicadas a problemas de estabilidade robusta de sistemas com incerteza politópica (Kau et al., 2005; Lin et al., 2006; Yang e Dong, 2008).

Vale a pena mencionar outros resultados tratando de estabilização não quadrática de sistemas nebulosos de T-S (Arrifano et al., 2006; Rhee e Won, 2006; Avellar et al., 2008; Mozelli, Palhares e Avellar, 2009). Quando limitantes da taxa de variação das funções de pertinência são conhecidos, funções de Lyapunov dependentes de parâmetros (também chamadas funções de Lyapunov nebulosas) podem ser usadas para diminuir o conservadorismo (Tanaka et al., 2007; Lam e Leung, 2007; Mozelli et al., 2008; Mozelli, Palhares e Avellar, 2009; Mozelli, Palhares, Souza e Mendes, 2009). Outras linhas emergentes no contexto de sistemas nebulosos de T-S utilizam funções de Lyapunov polinomiais no estado (Tanaka et al., 2009) e técnicas de otimização baseadas em soma de quadrados (Prajna et al., 2004).
O principal objetivo deste artigo é fornecer um procedimento sistemático para construir uma seqüência de LMIs (chamadas relaxações LMIs), com garantia de convergência, para resolver o problema de estabilizabilidade quadrática e de controle $\mathscr{H}_{2}$ e $\mathscr{H}_{\infty}$ por realimentação de estados para sistemas nebulosos de T-S. Como um primeiro passo, a condição de estabilizabilidade quadrática e as condições de estabilizabilidade quadrática com custo garantido $\mathscr{H}_{2}$ e $\mathscr{H}_{\infty}$ são descritas por LMIs dependentes de parâmetros com variáveis de folga (multiplicadores) provenientes do Lema de Finsler. As condições propostas fornecem ganhos de controle por realimentação de estados que são polinômios homogêneos de grau $g$ arbitrário, e contêm o controlador de grau $g=1 \mathrm{em}$ $\alpha$ (isto é, o compensador paralelo distribuído, linearmente dependente de $\alpha$ ) como caso particular. Para resolver essas LMIs dependentes de parâmetros com variáveis de folga e variáveis de decisão de grau arbitrário, são propostas relaxações baseadas no Teorema de Pólya e em propriedades de parâmetros pertencentes ao simplex unitário que resultam em uma seqüência de LMIs. Essas LMIs são cada vez menos conservadoras com o aumento do nível de relaxação, produzindo uma realimentação de estados dependente de $\alpha$ de grau $g$ sempre que tal controlador existir. Além disso, no caso do controle $\mathscr{H}_{2}$ e $\mathscr{H}_{\infty}$, as LMIs propostas convergem assintoticamente para os valores ótimos de custo garantido $\mathscr{H}_{2}$ e $\mathscr{H}_{\infty}$ sob a estabilidade quadrática. O principal apelo das condições propostas é a eficiência numérica, corroborada aqui por meio de exemplos que mostram que, com esforço computacional comparável, é possível obter resultados melhores do que os de condições disponíveis na literatura.

\section{FORMULAÇÃO DO PROBLEMA}

Seja a $i$-ésima regra de um modelo nebuloso de T-S dada por (veja por exemplo Tanaka et al. (1998a), Tanaka e Wang (2001) e Tuan et al. (2001) para detalhes)

$$
\begin{aligned}
& \mathrm{SE} z_{1}(t) \text { É } N_{i 1} \mathrm{E} \ldots \mathrm{E} z_{\ell}(t) \text { É } N_{i \ell} \\
& \text { ENTÃO }\left\{\begin{array}{l}
\dot{x}(t)=A_{i} x(t)+B_{i} u(t)+E_{i} w(t) \\
y(t)=C_{i} x(t)+D_{i} u(t)+F_{i} w(t)
\end{array}\right.
\end{aligned}
$$

em que $x(t) \in \mathbb{R}^{n}$ é o estado, $u(t) \in \mathbb{R}^{m}$ é a entrada de controle, $w(t) \in \mathbb{R}^{q}$ é uma entrada exógena e $y(t) \in \mathbb{R}^{p}$ é a saída controlada. As matrizes dos subsistemas lineares, conhecidas a priori, são dadas por $A_{i} \in \mathbb{R}^{n \times n}$, $B_{i} \in \mathbb{R}^{n \times m}, E_{i} \in \mathbb{R}^{n \times q}, C_{i} \in \mathbb{R}^{p \times n}, D_{i} \in \mathbb{R}^{p \times m}$ e $F_{i} \in \mathbb{R}^{p \times q}$, $i=1, \ldots, N$. As variáveis de premissa (assumidas como independentes de $u(t))$ são $z_{1}(t), \ldots, z_{\ell}(t), N_{i j}$ são conjuntos nebulosos e $M_{i j}\left(z_{i}(t)\right)$ é o grau de pertinência de $z_{i}(t)$ em $N_{i j}$. Normalizando o peso $w_{i}(t)=\prod_{j=1}^{\ell} N_{i j}\left(z_{i}(t)\right)$ de 
cada regra por

$$
\alpha_{i}(t)=\frac{w_{i}(t)}{\sum_{j=1}^{N} w_{j}(t)}, i=1, \ldots, N
$$

em que $\alpha(t)=\left[\begin{array}{lll}\alpha_{1}(t) & \ldots & \alpha_{N}(t)\end{array}\right]^{\prime}$ pertence ao simplex unitário

$$
\Lambda_{N}=\left\{\lambda \in \mathbb{R}^{N}: \sum_{i=1}^{N} \lambda_{i}=1, \lambda_{i} \geq 0, i=1, \ldots, N\right\}
$$

para todo $t \geq 0$, tem-se que o sistema nebuloso de T-S pode ser representado por um modelo politópico variante no tempo dado por

$$
\begin{aligned}
& \dot{x}(t)=A(\alpha(t)) x(t)+B(\alpha(t)) u(t)+E(\alpha(t)) w(t) \\
& y(t)=C(\alpha(t)) x(t)+D(\alpha(t)) u(t)+F(\alpha(t)) w(t)
\end{aligned}
$$

com

$$
\begin{aligned}
&(A, B, C, D, E, F)(\alpha(t)) \\
& \quad=\sum_{i=1}^{N} \alpha_{i}(t)\left(A_{i}, B_{i}, C_{i}, D_{i}, E_{i}, F_{i}\right), \alpha(t) \in \Lambda_{N}
\end{aligned}
$$

Considere uma lei de controle por realimentação de estados com ganhos dependentes de parâmetros dada por

$$
u(t)=K(\alpha(t)) x(t)
$$

que permite escrever o sistema em malha fechada como

$$
\begin{aligned}
& \dot{x}(t)=A_{c l}(\alpha(t)) x(t)+E(\alpha(t)) w(t) \\
& y(t)=C_{c l}(\alpha(t)) x(t)+F(\alpha(t)) w(t)
\end{aligned}
$$

com

$$
\begin{aligned}
& A_{c l}(\alpha(t)) \triangleq A(\alpha(t)+B(\alpha(t)) K(\alpha(t)), \\
& C_{c l}(\alpha(t)) \triangleq C(\alpha(t))+D(\alpha(t)) K(\alpha(t))
\end{aligned}
$$

Note que para $K(\alpha(t))$ linearmente dependente de parâmetros, isto é,

$$
K(\alpha(t))=\sum_{i=1}^{N} \alpha_{i}(t) K_{i}, K_{i} \in \mathbb{R}^{m \times n}
$$

tem-se o caso particular de um compensador paralelo distribuído, intensamente investigado na literatura. Na realimentação por compensador paralelo distribuído, uma regra de controle $u(t)=K_{i} x(t)$ é aplicada à correspondente $i$-ésima regra do modelo nebuloso de T-S (2). O controlador $K(\alpha(t))$ sintetizado com as condições propostas neste trabalho é uma função polinomial de grau $g$ arbitrário no parâmetro variante no tempo $\alpha(t)$, sendo portanto mais geral do que o compensador paralelo distribuído (11), caso ao qual se reduz quando o polinômio considerado for de grau $g=1$.

Como usualmente ocorre em sistemas descritos por modelos nebulosos de T-S, para a implementação em tempo real é preciso identificar ou medir as variáveis de premissa variantes no tempo $z_{i}(t), i=1, \ldots, N$, determinar o vetor $\alpha(t)$ e então construir $K(\alpha(t))$ para aplicar a lei de controle $u(t)$.

A próxima seção apresenta três lemas que descrevem condições convexas para o cômputo do ganho $K(\alpha(t))$ que estabiliza quadraticamente o sistema em malha fechada, levando em conta apenas estabilização ou a estabilização com minimização dos custos garantidos $\mathscr{H}_{2}$ e $\mathscr{H}_{\infty}$. As condições apresentadas a seguir utilizam variáveis de folga provenientes do Lema de Finsler (de Oliveira e Skelton, 2001), que contribuem para uma maior eficiência numérica das relaxações propostas na Seção 5 para a resolução das LMIs dependentes de parâmetros. Por simplicidade, $\alpha(t)$ será doravante denotado $\alpha$.

\section{SÍNTESE COM VARIÁVEIS DE FOLGA}

\subsection{Estabilizabilidade quadrática}

Lema 1 Existe um ganho $K(\alpha) \in \mathbb{R}^{m \times n}$ que estabiliza quadraticamente o sistema nebuloso de T-S (9)-(10) se e somente se existirem uma matriz simétrica definida positiva $W \in \mathbb{R}^{n \times n}$ e matrizes $Z(\alpha) \in \mathbb{R}^{m \times n}$ e $\mathscr{X}(\alpha) \in$ $\mathbb{R}^{2 n \times n}$ tais que

$$
\mathscr{L}(\alpha) \triangleq \mathscr{Q}(\alpha)+\mathscr{X}(\alpha) \mathscr{B}(\alpha)+\mathscr{B}(\alpha)^{\prime} \mathscr{X}(\alpha)^{\prime}<\mathbf{0}_{2 n}
$$

$\operatorname{com}^{1}$

$$
\begin{gathered}
\mathscr{Q}(\alpha)=\left[\begin{array}{cc}
B(\alpha) Z(\alpha)+Z(\alpha)^{\prime} B(\alpha)^{\prime} & W \\
\star & \mathbf{0}_{n}
\end{array}\right], \\
\mathscr{B}(\alpha)=\left[\begin{array}{ll}
A(\alpha)^{\prime} & -\mathbf{I}_{n}
\end{array}\right]
\end{gathered}
$$

Prova: Primeiramente, note que $\mathscr{B}(\alpha)^{\perp}=\left[\begin{array}{ll}\mathbf{I}_{n} & A(\alpha)\end{array}\right]^{\prime} \mathrm{e}$ que $\mathscr{B}(\alpha) \mathscr{B}(\alpha)^{\perp}=\mathbf{0}$. Do Lema de Finsler (de Oliveira e Skelton, 2001), que estabelece a equivalência

$$
\mathscr{B}^{\perp^{\prime}} \mathscr{Q} \mathscr{B}^{\perp}<\mathbf{0} \Leftrightarrow \exists \mathscr{X}: \mathscr{Q}+\mathscr{X} \mathscr{B}+\mathscr{B}^{\prime} \mathscr{X}^{\prime}<\mathbf{0}
$$

tem-se que (12) é equivalente a

$$
\begin{aligned}
\mathbf{0}_{n} & >\mathscr{B}(\alpha)^{\perp^{\prime}} \mathscr{Q}(\alpha) \mathscr{B}(\alpha)^{\perp} \\
& =\left[\begin{array}{c}
\mathbf{I}_{n} \\
A(\alpha)^{\prime}
\end{array}\right]^{\prime}\left[\begin{array}{cc}
B(\alpha) Z(\alpha)+Z(\alpha)^{\prime} B(\alpha)^{\prime} & W \\
\star & \mathbf{0}_{n}
\end{array}\right]\left[\begin{array}{c}
\mathbf{I}_{n} \\
A(\alpha)^{\prime}
\end{array}\right] \\
& =A(\alpha) W+W A(\alpha)^{\prime}+B(\alpha) Z(\alpha)+Z(\alpha)^{\prime} B(\alpha)^{\prime}
\end{aligned}
$$

\footnotetext{
${ }^{1} \mathrm{O}$ símbolo $\star$ representa blocos simétricos nas LMIs.
} 
que, sob a transformação de variáveis (Bernussou et al., 1989)

$$
K(\alpha)=Z(\alpha) W^{-1}
$$

é uma condição necessária e suficiente para a estabilizabilidade quadrática do sistema com ganhos de realimentação de estados dependentes de parâmetros (Montagner, Oliveira, Peres e Bliman, 2007).

Várias condições para a obtenção de $W=W^{\prime}>0$ e $Z(\alpha)$ que garantam que (13) é verificada para todo $\alpha \in \Lambda_{N}$ podem ser encontradas na literatura, tanto no contexto de sistemas nebulosos de T-S (Kim e Lee, 2000; Tanaka e Wang, 2001; Teixeira et al., 2003; Liu e Zhang, 2003; Fang et al., 2006) quanto no caso de estabilizabilidade quadrática por ganhos escalonados para sistemas contínuos variantes no tempo (Apkarian e Gahinet, 1995; Apkarian et al., 1995; Scherer, 2001; Wang e Balakrishnan, 2002; Montagner e Peres, 2006; Montagner, Oliveira, Peres e Bliman, 2007). Sabe-se que a solução do problema pode ser restrita, sem perda de generalidade, a matrizes $Z(\alpha)$ que sejam lineares em $\alpha$. Tal propriedade decorre do Lema de Finsler (de Oliveira e Skelton, 2001), tendo sido provada por exemplo em Tuan et al. (2001) no contexto de sistemas nebulosos. Veja também Montagner, Oliveira, Peres e Bliman (2007, Corolário 1).

Para encontrar $W=W^{\prime}>0$ e $Z(\alpha)$ solução de (13), resultados clássicos como os de Tanaka et al. (1998a) expressam a desigualdade como uma forma quadrática em $\alpha$ com coeficientes que são funções matriciais lineares. Impor a negatividade de todos os coeficientes é suficiente para garantir que a desigualdade (13) é verificada, mas muitas vezes é bastante conservador, pois nem todos os termos de uma soma precisam ser negativos para que a soma seja menor que zero. Relaxações menos conservadoras, porém ainda suficientes, como as de Kim e Lee (2000), exploram o uso de variáveis de folga no lado direito das desigualdades matriciais lineares. Outros resultados foram propostos, como as condições de Liu e Zhang (2003), Teixeira et al. (2003) e Fang et al. (2006), mas nenhuma com convergência garantida para a solução do problema. Recentemente, relaxações oriundas do Teorema de Pólya, que conduzem a condições necessárias e suficientes para a estabilizabilidade quadrática, foram investigadas em Montagner, Oliveira e Peres (2007) e Sala e Ariño (2007).

Embora os trabalhos de Montagner, Oliveira e Peres (2007) e Sala e Ariño (2007) forneçam relaxações convergentes para a solução do problema da estabilizabilidade quadrática com $K(\alpha)$ de grau um, é possível aumentar a eficiência numérica das condições em termos de estabilidade numérica dos testes e de convergência para um controlador que resolva o problema da estabiliza- bilidade com um menor custo computacional associado usando-se variáveis de folga, provenientes do Lema de Finsler (Montagner, Oliveira e Peres, 2007) ou colocadas em um polinômio de grau arbitrário a determinar que majora o lado direito de (13) e ao qual se impõe a negatividade (Sala e Ariño, 2007). Uma comparação entre as duas estratégias em termos de complexidade e das possíveis extensões pode ser encontrada em Montagner et al. (2009).

As relaxações propostas neste trabalho, dadas na Seção 5, exploram o uso de variáveis de folga advindas do Lema de Finsler, como descrito no Lema 1, generalizadas para a obtenção de um ganho $K(\alpha)$ polinomial homogêneo em $\alpha \in \Lambda_{N}$ com grau $g \geq 1$. Sabe-se que, se houver solução para as LMIs dependentes de parâmetros do Lema 1, então existe uma solução dada por matrizes polinomiais homogêneas (Bliman et al., 2006). Assim, $Z(\alpha)$ e $\mathscr{X}(\alpha)$ podem ser restritas a funções polinomiais homogêneas de grau $g$ em $\alpha$ e, conseqüentemente, o ganho $K(\alpha)$ será polinomial homogêneo de grau $g$. Se por um lado a solução do Lema 1 pode ser restrita a matrizes $Z(\alpha)$ e $\mathscr{X}(\alpha)$ lineares em $\alpha$, sem perda de generalidade, o custo computacional associado às relaxações de Pólya para obter essas matrizes pode ser elevado. Por outro lado, se existir solução de grau um para o Lema 1, sempre haverá solução para grau mais elevado (Bliman et al., 2006). Assim, buscando-se $Z(\alpha)$ e $\mathscr{X}(\alpha)$ de graus maiores, pode-se convergir para a solução do problema com menor esforço computacional e menores valores nas relaxação de Pólya, conforme ilustram os exemplos numéricos da Seção 6 .

\subsection{Custo $\mathscr{H}_{\infty}$}

O custo garantido $\mathscr{H}_{\infty}$ é um índice de desempenho relacionado com a capacidade de o sistema em malha fechada rejeitar perturbações do tipo sinais de energia (Boyd et al., 1994). O sistema nebuloso de T-S (9)-(10) tem um custo garantido $\mathscr{H}_{\infty}$ dado por $\gamma>0$ sob estabilidade quadrática se, além de ser quadraticamente estável,

$$
\|y(t)\|_{2} \leq \gamma\|w(t)\|_{2}, \quad \forall \alpha \in \Lambda_{N}
$$

para todo $w(t) \in \mathscr{L}_{2}$. Nesse caso, diz-se que o sistema (9)-(10) é quadraticamente estável com um custo garantido $\mathscr{H}_{\infty}$ dado por $\gamma>0$ (Tanaka e Wang, 2001).

O próximo lema fornece condições de dimensão infinita (em termos de $\alpha \in \Lambda_{N}$ ) para computar um ganho $K(\alpha)$ que assegura a estabilidade quadrática do sistema em malha fechada com um custo $\mathscr{H}_{\infty}$ dado por $\gamma$.

Lema 2 Existe um ganho $K(\alpha) \in \mathbb{R}^{m \times n}$ que estabiliza quadraticamente o sistema nebuloso de T-S (9)-(10) com 
um custo garantido $\mathscr{H}_{\infty}$ dado por $\gamma>0$ se e somente se existirem uma matriz simétrica definida positiva $W \in$ $\mathbb{R}^{n \times n}$ e matrizes $Z(\alpha) \in \mathbb{R}^{m \times n}$ e $\mathscr{X}(\alpha) \in \mathbb{R}^{(2 n+p+q) \times(n+q)}$ tais que

$$
\mathscr{Q}(\alpha)+\mathscr{X}(\alpha) \mathscr{B}(\alpha)+\mathscr{B}(\alpha)^{\prime} \mathscr{X}(\alpha)^{\prime}<\mathbf{0}_{2 n+p+q}
$$

com

$$
\begin{aligned}
& \mathscr{Q}(\alpha)=\left[\begin{array}{cc}
B(\alpha) Z(\alpha)+Z(\alpha)^{\prime} B(\alpha)^{\prime} & W \\
\star & \mathbf{0}_{n} \\
\star & \star \\
\star & \star
\end{array}\right. \\
& \left.\begin{array}{cc}
\mathbf{0}_{n} & Z(\alpha)^{\prime} D(\alpha)^{\prime} \\
\mathbf{0}_{n \times q} & \mathbf{0}_{n \times p} \\
\mathbf{I}_{q} & \mathbf{0}_{q \times p} \\
\star & -\gamma^{2} \mathbf{I}_{p}
\end{array}\right] \\
& \mathscr{B}(\alpha)=\left[\begin{array}{cccc}
A(\alpha)^{\prime} & -\mathbf{I}_{n} & \mathbf{0}_{n \times q} & C(\alpha)^{\prime} \\
E(\alpha)^{\prime} & \mathbf{0}_{q \times n} & -\mathbf{I}_{q} & F(\alpha)^{\prime}
\end{array}\right]
\end{aligned}
$$

seja satisfeita para todo $\alpha \in \Lambda_{N}$. No caso afirmativo, o ganho $K(\alpha)$ é dado por (14).

Prova: Primeiro, note que $\mathscr{B}(\alpha) \mathscr{B}(\alpha)^{\perp}=\mathbf{0}$ com

$$
\mathscr{B}(\alpha)^{\perp^{\prime}}=\left[\begin{array}{cccc}
\mathbf{I}_{n} & A(\alpha) & E(\alpha) & \mathbf{0}_{n \times p} \\
\mathbf{0}_{p \times n} & C(\alpha) & F(\alpha) & \mathbf{I}_{p}
\end{array}\right]
$$

Pelo Lema de Finsler (de Oliveira e Skelton, 2001), (16) é equivalente a $\mathscr{B}(\alpha)^{\perp^{\prime}} \mathscr{Q}(\alpha) \mathscr{B}(\alpha)^{\perp}<\mathbf{0}$, isto é,

$$
\begin{gathered}
\mathscr{B}(\alpha)^{\perp^{\prime}} \mathscr{Q}(\alpha) \mathscr{B}(\alpha)^{\perp} \\
=\left[\begin{array}{c}
A(\alpha) W+W A(\alpha)^{\prime}+B(\alpha) Z(\alpha) \\
+Z(\alpha)^{\prime} B(\alpha)^{\prime}+E(\alpha) E(\alpha)^{\prime} \\
\star \\
E(\alpha) F(\alpha)^{\prime}+W C(\alpha)^{\prime}+Z(\alpha)^{\prime} D(\alpha)^{\prime} \\
F(\alpha) F(\alpha)^{\prime}-\gamma^{2} \mathbf{I}_{p}
\end{array}\right] \\
=\left[\begin{array}{c}
A_{c l}(\alpha) W+W A_{c l}(\alpha)^{\prime}+E(\alpha) E(\alpha)^{\prime} \\
\star \\
E(\alpha) F(\alpha)^{\prime}+W C_{c l}(\alpha)^{\prime} \\
F(\alpha) F(\alpha)^{\prime}-\gamma^{2} \mathbf{I}_{p}
\end{array}\right]<\mathbf{0}_{n+p}
\end{gathered}
$$

A desigualdade acima é conhecida na literatura (para um valor fixo de $\alpha \in \Lambda_{N}$ ) como bounded real lemma (Boyd et al., 1994) e, se verificada para todo $\alpha \in \Lambda_{N}$, assegura (15).

Note que o Lema 2 é formulado em termos de LMIs dependentes de parâmetros pertencentes ao simplex unitário e, pelas mesmas razões expostas anteriormente, as matrizes $Z(\alpha)$ e $\mathscr{X}(\alpha)$ no Lema 2 podem ser restritas a matrizes polinomiais homogêneas de grau $g$ em $\alpha$, sem perda de generalidade. Portanto, o ganho $K(\alpha)$ pode ser restrito sem perda de generalidade a um ganho polinomial homogêneo de grau $g$ em $\alpha$. O caso do compensador paralelo distribuído é obtido quando $g=1$.

Como $\gamma$ está relacionado ao nível de atenuação de perturbações, é de grande interesse determinar um controlador que assegure a estabilidade com um mínimo valor de $\gamma$. O mínimo global para $\gamma$, sob as condições do Lema 2, é obtido por meio do problema convexo de otimização

$$
\begin{array}{r}
\gamma_{m}=\min \gamma \\
\text { s.a }(16)
\end{array}
$$

\subsection{Custo $\mathscr{H}_{2}$}

Da mesma forma que no caso do controle $\mathscr{H}_{\infty}$, podese enunciar o problema do cômputo de um ganho $K(\alpha)$ que estabilize quadraticamente o sistema nebuloso de T-S (9)-(10) com um custo garantido $\mathscr{H}_{2}$, seguindo a formulação do problema de projeto de controladores $\mathscr{H}_{2}$ por realimentação de estados para sistemas variantes no tempo (de Souza et al., 2003; Green e Limebeer, 1995). $\mathrm{Na}$ determinação do ganho $\mathscr{H}_{2}$, assume-se que o termo de transmissão direta no modelo nebuloso de T-S (9)(10) é nulo (isto é, $F(\alpha)=\mathbf{0}$ ), para assegurar a existência de custos finitos. O lema a seguir estabelece a relação entre a existência de um ganho $K(\alpha)$ quadraticamente estabilizante com custo garantido $\mathscr{H}_{2}$ e a solução de uma LMI dependente de parâmetros.

Lema 3 Existe um ganho $K(\alpha) \in \mathbb{R}^{m \times n}$ que estabiliza quadraticamente o sistema nebuloso de T-S (9)-(10) com um custo garantido $\mathscr{H}_{2}$ dado por $\rho>0$ se e somente se existirem uma matriz simétrica definida positiva $W \in \mathbb{R}^{n \times n}$, uma matriz simétrica $M(\alpha) \in \mathbb{R}^{p \times p}$ e matrizes $Z(\alpha) \in \mathbb{R}^{m \times n}$ e $\mathscr{X}(\alpha) \in \mathbb{R}^{(2 n+q) \times(n+q)}$ tais que

$$
\begin{gathered}
\operatorname{Tr}(M(\alpha))<\rho^{2} \\
\mathscr{T}(\alpha) \triangleq\left[\begin{array}{ccc}
W & W C(\alpha)^{\prime}+Z(\alpha)^{\prime} D(\alpha)^{\prime} \\
\star & M(\alpha)
\end{array}\right]>\mathbf{0}_{n+p} \\
{\left[\begin{array}{ccc}
B(\alpha) Z(\alpha)+Z(\alpha)^{\prime} B(\alpha)^{\prime} & W & \mathbf{0}_{n \times q} \\
\star & \mathbf{0}_{n} & \mathbf{0}_{n \times q} \\
\star & \star & \mathbf{I}_{q}
\end{array}\right]} \\
+\mathscr{X}(\alpha) \mathscr{B}(\alpha)+\mathscr{B}(\alpha)^{\prime} \mathscr{X}(\alpha)^{\prime}<\mathbf{0}_{2 n+q} \\
\mathscr{B}(\alpha)=\left[\begin{array}{ccc}
A(\alpha)^{\prime} & -\mathbf{I}_{n} & \mathbf{0}_{n \times q} \\
E(\alpha)^{\prime} & \mathbf{0}_{q \times n} & -\mathbf{I}_{q}
\end{array}\right]
\end{gathered}
$$

sejam satisfeitas para todo $\alpha \in \Lambda_{N}$. Em caso positivo, os ganhos do controlador são dados por (14). 
Prova: Pré e pós-multiplicando (20) por $\mathscr{B}(\alpha)^{\perp^{\prime}}$ e $\mathscr{B}(\alpha)^{\perp}$, respectivamente, com

$$
\mathscr{B}(\alpha)^{\perp^{\prime}}=\left[\begin{array}{lll}
\mathbf{I}_{n} & A(\alpha) & E(\alpha)
\end{array}\right], \mathscr{B}(\alpha) \mathscr{B}(\alpha)^{\perp}=\mathbf{0}
$$

tem-se que (20) implica em

$$
\begin{aligned}
A(\alpha) W+W A(\alpha)^{\prime}+ & B(\alpha) Z(\alpha) \\
& +Z(\alpha)^{\prime} B(\alpha)^{\prime}+E(\alpha) E(\alpha)^{\prime}<\mathbf{0}_{n}
\end{aligned}
$$

ou, $\operatorname{com} K(\alpha)=Z(\alpha) W^{-1}$, em

$$
A_{c l}(\alpha) W+W A_{c l}(\alpha)^{\prime}+E(\alpha) E(\alpha)^{\prime}<\mathbf{0}_{n}
$$

A volta (ou seja, se (21) é verificada então (20) também é válida) pode ser provada de maneira similar à da prova do Lema 2, utilizando-se o Lema de Finsler (de Oliveira e Skelton, 2001). Das condições (18)-(19) tem-se por complemento de Schur (Boyd et al., 1994) que

$$
\rho^{2}>\operatorname{Tr}(M(\alpha)) \geq \operatorname{Tr}\left(C_{c l}(\alpha) W C_{c l}(\alpha)^{\prime}\right)
$$

e, portanto, recuperam-se as condições de de Souza et al. (2003) e Green e Limebeer (1995) que asseguram estabilidade quadrática com custo garantido $\mathscr{H}_{2}$ dado por $\rho$.

Assim como no caso $\mathscr{H}_{\infty}$, o controlador que assegura a estabilidade quadrática com um mínimo valor de $\rho$ é obtido por meio do problema convexo de otimização

$$
\begin{array}{r}
\rho_{m}=\min \rho \\
\text { s.a }(18)-(20)
\end{array}
$$

Como nos lemas anteriores, o Lema 3 é descrito por LMIs dependentes de parâmetros, tendo por solução geral, além da matriz $W$ associada à estabilidade quadrática, matrizes polinomiais homogêneas $M(\alpha), Z(\alpha)$ e $\mathscr{X}(\alpha)$. Uma breve descrição de matrizes polinomiais homogêneas e da notação utilizada no resto do artigo é dada na próxima seção. Maiores detalhes sobre a caracterização da solução de LMIs com parâmetros no simplex unitário podem ser obtidos em Oliveira e Peres (2007).

\section{MATRIZES POLINOMIAIS}

Uma matriz polinomial homogênea de grau $g$ pode ser escrita de forma geral como

$$
\begin{aligned}
Q(\alpha)=\sum_{k \in \mathscr{K}(g)} \alpha^{k} Q_{k}, \quad \alpha^{k}=\alpha_{1}^{k_{1}} \alpha_{2}^{k_{2}} \cdots \alpha_{N}^{k_{N}}, & \\
k & =k_{1} k_{2} \cdots k_{N}
\end{aligned}
$$

sendo que $\alpha_{1}^{k_{1}} \alpha_{2}^{k_{2}} \cdots \alpha_{N}^{k_{N}}, \alpha \in \Lambda_{N}, k_{i} \in \mathbb{N}, i=1, \ldots, N$ são os monômios e $Q_{k} \in \mathbb{R}^{n \times n}, \forall k \in \mathscr{K}(g)$ são coeficientes matriciais. Por definição, $\mathscr{K}(g)$ é o conjunto de ênuplas obtidas a partir de todas as combinações de inteiros não negativos $k_{i}, i=1, \ldots, N$, tais que $k_{1}+k_{2}+\ldots+k_{N}=g$, e $N$ é o número de regras do modelo nebuloso de T-S.

Por exemplo, para um polinômio homogêneo de grau $g=2 \operatorname{com} N=2$, tem-se $\mathscr{K}(2)=\{02,11,20\}$, correspondendo na forma matricial a $Q(\alpha)=\alpha_{2}^{2} Q_{02}+\alpha_{1} \alpha_{2} Q_{11}+$ $\alpha_{1}^{2} Q_{20}$. Matrizes constantes (grau zero) são obtidas por meio de (23), para $g=0$.

Por definição, para ênuplas $k, k^{\prime}$ pode-se escrever $k \succeq k^{\prime}$ se $k_{i} \geq k_{i}^{\prime}, i=1, \ldots, N$. Operações de soma $k+k^{\prime}$ e subtração $k-k^{\prime}$ (sempre que $k \succeq k^{\prime}$ ) são definidas elemento a elemento. Considere também as seguintes definições para a ênupla $e_{i}$ e o coeficiente $\pi(k)$

$$
e_{i}=0 \cdots 0 \underbrace{1}_{\text {i-ésimo }} 0 \cdots 0, \pi(k)=\left(k_{1} !\right)\left(k_{2} !\right) \cdots\left(k_{N} !\right)
$$

Usando as notações definidas acima, relaxações LMIs convergentes para a solução dos lemas 1,2 e 3 são fornecidas na próxima seção.

\section{RELAXAÇÕES PROPOSTAS}

Teorema 1 O sistema nebuloso de T-S (9)-(10) é quadraticamente estabilizável se e somente se existirem uma matriz simétrica definida positiva $W \in \mathbb{R}^{n \times n}$, matrizes $Z_{k} \in \mathbb{R}^{m \times n}, X_{k} \in \mathbb{R}^{2 n \times n}, k \in \mathscr{K}(g)$, um grau $g \geq 1, g \in \mathbb{N}$, e um $d \in \mathbb{N}$ suficientemente grande tais que

$$
\begin{aligned}
L_{k}= & \sum_{\substack{k^{\prime} \in \mathscr{K}(d) \\
k \succeq k^{\prime}}}\left(\sum _ { \substack { i \in \{ 1 , \ldots , N \} \\
k _ { i } > k _ { i } ^ { \prime } } } \frac { d ! } { \pi ( k ^ { \prime } ) } \left(\mathscr{Q}_{k-k^{\prime}-e_{i}}+X_{k-k^{\prime}-e_{i}} \mathscr{B}_{i}\right.\right. \\
& \left.\left.+\mathscr{B}_{i}^{\prime} X_{k-k^{\prime}-e_{i}}^{\prime}\right)\right)<\mathbf{0}_{2 n}, \quad \forall k \in \mathscr{K}(g+d+1)
\end{aligned}
$$

com

$\mathscr{Q}_{k-k^{\prime}-e_{i}}=\left[\begin{array}{cc}B_{i} Z_{k-k^{\prime}-e_{i}}+Z_{k-k^{\prime}-e_{i}}^{\prime} B_{i}^{\prime} & \beta W \\ \beta W & \mathbf{0}_{n}\end{array}\right], \mathscr{B}_{i}=\left[\begin{array}{ll}A_{i}^{\prime} & -\mathbf{I}_{n}\end{array}\right]$

e $\beta=g !\left(k_{i}-k_{i}^{\prime}\right) / \pi\left(k-k^{\prime}\right)$. No caso afirmativo, o controlador nebuloso polinomial homogêneo de grau $g$ que estabiliza quadraticamente o sistema é dado por

$$
K(\alpha)=\sum_{k \in \mathscr{K}(g)} \alpha^{k} Z_{k} W^{-1}
$$

Prova: Primeiro note que considerando $\mathscr{X}(\alpha)$ e $Z(\alpha)$ como matrizes polinomiais homogêneas de grau $g$, pode- 
se reescrever o lado esquerdo de (12) na forma equivalente

$$
\left(\alpha_{1}+\cdots+\alpha_{N}\right)^{d} \mathscr{L}(\alpha)=\sum_{k \in \mathscr{K}(g+d+1)} \alpha^{k} L_{k}
$$

para quaisquer $d \in \mathbb{N}$ e $1 \leq g \in \mathbb{N}$ uma vez que $\left(\alpha_{1}+\right.$ $\left.\cdots+\alpha_{N}\right)=1$. Para $d=0, L_{k}$ são os coeficientes do polinômio homogêneo $\mathscr{L}(\alpha)$ de grau $g+1$. Se $L_{k}<\mathbf{0}_{2 n}$ para qualquer $g>1$ e para todo $k \in \mathscr{K}(g+1)$, então o polinômio $\mathscr{L}(\alpha)$ é definido negativo, concluindo a suficiência da prova. Para provar a necessidade, note que se $\mathscr{L}(\alpha)<\mathbf{0}_{2 n}$ para todo $\alpha \in \Lambda_{N}$, então do teorema de Pólya (Hardy et al., 1952; Scherer, 2005; Powers e Reznick, 2001) tem-se que também existe um polinômio homogêneo, possivelmente de grau maior, com todos os coeficientes definidos negativos. Tal polinômio é sempre obtido usando-se o lado esquerdo de (27) com um $d$ suficientemente grande.

O nível de relaxação $d$ (relaxação de Pólya) é a chave para construir uma seqüência de LMIs convergentes. O aumento em $d$ reduz o conservadorismo gerado pela imposição de que todos os coeficientes do polinômio homogêneo sejam definidos negativos para que o polinômio seja definido negativo para todo $\alpha \in \Lambda_{N}$. Por exemplo, considere $N=2, g=1$, e $d=0$. As LMIs resultantes são

$$
\begin{aligned}
L_{20}= & \mathscr{Q}_{10}+X_{10} \mathscr{B}_{1}+\mathscr{B}_{1}^{\prime} X_{10}^{\prime}<\mathbf{0}_{2 n}, \\
L_{02}= & \mathscr{Q}_{01}+X_{01} \mathscr{B}_{2}+\mathscr{B}_{2}^{\prime} X_{01}^{\prime}<\mathbf{0}_{2 n}, \\
L_{11}= & \mathscr{Q}_{10}+\mathscr{Q}_{01}+X_{10} \mathscr{B}_{2}+\mathscr{B}_{2}^{\prime} X_{10}^{\prime} \\
& +X_{01} \mathscr{B}_{1}+\mathscr{B}_{1}^{\prime} X_{01}^{\prime}<\mathbf{0}_{2 n}
\end{aligned}
$$

As condições $L_{20}<\mathbf{0}_{2 n}$ e $L_{02}<\mathbf{0}_{2 n}$ são, de fato, também necessárias (cada modelo linear deve ser estabilizável). A condição $L_{11}<\mathbf{0}_{2 n}$ (termo cruzado), entretanto, é apenas suficiente. Para $d=1$, as LMIs resultantes

$L_{20}<\mathbf{0}_{2 n}, \quad L_{02}<\mathbf{0}_{2 n}, \quad L_{11}+L_{20}<\mathbf{0}_{2 n}, \quad L_{11}+L_{02}<\mathbf{0}_{2 n}$

são mais relaxadas (note que nas LMIs acima não exigese que $L_{11}$ seja definido negativo). Esse é o princípio das relaxações de Pólya's e, se $\mathscr{L}(\alpha)<\mathbf{0}_{2 n}, \forall \alpha \in \Lambda_{N}$, então, para um $d$ suficientemente grande, todos os coeficientes $L_{k}$ serão definidos negativos. Note que o aumento em $d$ somente aumenta o número de LMIs, enquanto que o número de variáveis de decisão é mantido constante, pois só depende das dimensões do problema e do grau $g$ do ganho $K(\alpha)$ e dos multiplicadores $\mathscr{X}(\alpha)$. Se as condições do Teorema 1 são satisfeitas para um dado $d=\hat{d}$, então as condições também são satisfeitas para qualquer $d>\hat{d}$, uma vez que as desigualdades resultantes para $\hat{d}+1$ podem ser escritas como combinações positivas das desigualdades para $\hat{d}$. As afirmações acima são válidas para qualquer escolha de $g \geq 1$.
A estrutura do multiplicador $\mathscr{X}(\alpha)$ poderia ser restrita ao caso afim em $\alpha$ sem perda de generalidade, como discutido em Montagner, Oliveira, Peres e Bliman (2007, Corolário 1). O Teorema 1, entretanto, permite que $\mathscr{X}(\alpha)$ seja considerado como um polinômio homogêneo de grau arbitrário $g \geq 1$. O aumento de $g$ introduz mais variáveis de decisão (coeficientes de um polinômio homogêneo de grau $g$ ) no problema de otimização, melhorando a convergência das condições, pois pode-se chegar à necessidade com menores valores de $d$. Note também que as condições do Teorema 1 tornam-se cada vez menos conservadoras somente com o aumento de $g$, para um dado $d$. Adicionalmente, as condições do Teorema 1 convergem (i.e., são assintoticamente necessárias com o aumento de $d$ ) para qualquer $g \geq 1$. Além disso, estruturas polinomiais homogêneas de grau $g$ para o ganho $K(\alpha)$, embora mais complexas para ser implementadas, permitem em alguns casos o cômputo do ganho estabilizante com um menor esforço computacional. Finalmente, note que os graus do ganho polinomial $K(\alpha)$ e do multiplicador $\mathscr{X}(\alpha)$ poderiam ser distintos, permitindo maior flexibilidade nos procedimentos para a determinação da lei de controle estabilizante. A opção pelo mesmo grau $g$ simplifica as expressões das LMIs e produz bons resultados nos exemplos numéricos, sendo por isso mantida.

A seguir são apresentadas as relaxações para resolver os lemas 2 e 3 usando aproximações polinomiais homogêneas de grau $g$ para as matrizes $\mathscr{X}(\alpha)$ e $Z(\alpha)$. As provas, similares à prova do Teorema 1, não são apresentadas.

Teorema 2 O sistema nebuloso de T-S (9)-(10) é quadraticamente estabilizável com um custo garantido $\mathscr{H}_{\infty}$ dado por $\gamma>0$ se e somente se existirem uma matriz simétrica definida positiva $W \in \mathbb{R}^{n \times n}$, matrizes $Z_{k} \in \mathbb{R}^{m \times n}$, $X_{k} \in \mathbb{R}^{(2 n+p+q) \times(n+q)}, k \in \mathscr{K}(g)$, um grau $g \geq 1, g \in \mathbb{N}$, e um $d \in \mathbb{N}$ suficientemente grande tais que

$$
\begin{aligned}
& \sum_{\substack{k^{\prime} \in \mathscr{K}(d) \\
k \succeq k^{\prime}}}\left(\sum _ { \substack { i \in \{ 1 , \ldots , N \} \\
k _ { i } > k _ { i } ^ { \prime } } } \frac { d ! } { \pi ( k ^ { \prime } ) } \left(\mathscr{Q}_{k-k^{\prime} e_{i}}+X_{k-k^{\prime}-e_{i}} \mathscr{B}_{i}\right.\right. \\
& \left.+\mathscr{B}_{i}^{\prime} X_{k-k^{\prime}-e_{i}}^{\prime}\right)<\mathbf{0}_{2 n+p+q}, \quad \forall k \in \mathscr{K}(g+d+1)
\end{aligned}
$$


com

$$
\begin{aligned}
& \mathscr{Q}_{k-k^{\prime}-e_{i}}=\left[\begin{array}{cc}
B_{i} Z_{k-k^{\prime}-e_{i}}+Z_{k-k^{\prime}-e_{i}}^{\prime} B_{i}^{\prime} & \beta W \\
\star & \mathbf{0}_{n} \\
\star & \star \\
\star & \star
\end{array}\right. \\
& \left.\begin{array}{cc}
\mathbf{0}_{n} & Z_{k-k^{\prime}-e_{i}}^{\prime} D_{i}^{\prime} \\
\mathbf{0}_{n \times q} & \mathbf{0}_{n \times p} \\
\beta \mathbf{I}_{q} & \mathbf{0}_{q \times p} \\
\star & -\gamma^{2} \beta \mathbf{I}_{p}
\end{array}\right], \\
& \mathscr{B}_{i}=\left[\begin{array}{cccc}
A_{i}^{\prime} & -\mathbf{I}_{n} & \mathbf{0}_{n \times q} & C_{i}^{\prime} \\
E_{i}^{\prime} & \mathbf{0}_{q \times n} & -\mathbf{I}_{q} & F_{i}^{\prime}
\end{array}\right]
\end{aligned}
$$

e $\beta=g !\left(k_{i}-k_{i}^{\prime}\right) / \pi\left(k-k^{\prime}\right)$. No caso afirmativo, o controlador nebuloso polinomial homogêneo quadraticamente estabilizante de grau $g$ é dado por (26).

Corolário 1 Seja $\gamma^{*}$ o menor valor de $\gamma$ fornecido pelo Lema 2. Para qualquer $g \geq 1$, considere $\gamma^{*}(d)$ o menor valor de $\gamma$ fornecido pelo Teorema 2 para um dado $d$. Então, $\gamma^{*}(d)$ é uma função não crescente de $d$ e $\gamma^{*}(d) \rightarrow$ $\gamma^{*}$ quando $d \rightarrow+\infty$

Prova: Segue a prova do item (ii), de Montagner, Oliveira, Peres e Bliman (2007, Corolário 2).

Teorema 3 O sistema nebuloso de T-S (9)-(10) é quadraticamente estabilizável com um custo garantido $\mathscr{H}_{2}$ dado por $\rho>0$ se e somente se existirem uma matriz simétrica definida positiva $W \in \mathbb{R}^{n \times n}$, matrizes $Z_{k} \in \mathbb{R}^{m \times n}$, $M_{k} \in \mathbb{R}^{p \times p}, X_{k} \in \mathbb{R}^{(2 n+q) \times(n+q)}, k \in \mathscr{K}(g)$, um grau $g \geq 1$, $g \in \mathbb{N}$, e um $d \in \mathbb{N}$ suficientemente grande tais que

$$
\sum_{\substack{k^{\prime} \in \mathscr{K}(d) \\ k \succeq k^{\prime}}} \frac{d !}{\pi\left(k^{\prime}\right)}\left(\operatorname{Tr}\left(M_{k}\right)-\frac{g !}{\pi\left(k-k^{\prime}\right)} \rho^{2}\right) \leq 0, \quad \forall k \in \mathscr{K}(g+d)
$$

$$
\begin{aligned}
& \sum_{\substack{k^{\prime} \in \mathscr{K}(d) \\
k \succeq k^{\prime}}}\left(\sum_{\substack{i \in\{1, \ldots, N\} \\
k_{i}>k_{i}^{\prime}}} \frac{d !}{\pi\left(k^{\prime}\right)} \times\right. \\
& \left.\left[\begin{array}{cc}
\beta W & \beta W C_{i}^{\prime}+Z_{k-k^{\prime}-e_{i}}^{\prime} D_{i}^{\prime} \\
\star & M_{k-k^{\prime}-e_{i}}
\end{array}\right]\right)>\mathbf{0}_{n+p}, \forall k \in \mathscr{K}(g+d+1)
\end{aligned}
$$

$$
\begin{aligned}
& \sum_{\substack{k^{\prime} \in \mathscr{K}(d) \\
k \succeq k^{\prime}}}\left(\sum _ { \substack { i \in \{ 1 , \ldots , N \} \\
k _ { i } > k _ { i } ^ { \prime } } } \frac { d ! } { \pi ( k ^ { \prime } ) } \left(\mathscr{Q}_{k-k^{\prime} e_{i}}+X_{k-k^{\prime}-e_{i}} \mathscr{B}_{i}\right.\right. \\
& \left.\quad+\mathscr{B}_{i}^{\prime} X_{k-k^{\prime}-e_{i}}^{\prime}\right)<\mathbf{0}_{2 n+q}, \quad \forall k \in \mathscr{K}(g+d+1)
\end{aligned}
$$

com

$$
\begin{gathered}
\mathscr{Q}_{k-k^{\prime}-e_{i}}=\left[\begin{array}{cccc}
B_{i} Z_{k-k^{\prime}-e_{i}}+Z_{k-k^{\prime}-e_{i}}^{\prime} B_{i}^{\prime} & \beta W & \mathbf{0}_{n} \\
\star & & \mathbf{0}_{n} & \mathbf{0}_{n \times q} \\
\star & & \star & \beta \mathbf{I}_{q}
\end{array}\right], \\
\mathscr{B}_{i}=\left[\begin{array}{ccc}
A_{i}^{\prime} & -\mathbf{I}_{n} & \mathbf{0}_{n \times q} \\
E_{i}^{\prime} & \mathbf{0}_{q \times n} & -\mathbf{I}_{q}
\end{array}\right]
\end{gathered}
$$

e $\beta=g !\left(k_{i}-k_{i}^{\prime}\right) / \pi\left(k-k^{\prime}\right)$. No caso afirmativo, o controlador nebuloso polinomial homogêneo quadraticamente estabilizante de grau $g$ é dado por (26).

Corolário 2 Seja $\rho^{*}$ o menor valor de $\gamma$ fornecido pelo Lema 3. Para qualquer $g \geq 1$, considere $\rho^{*}(d)$ o menor valor de $\rho$ fornecido pelo Teorema 3 para um dado $d$. Então, $\rho^{*}(d)$ é uma função não crescente de $d$ e $\rho^{*}(d) \rightarrow$ $\rho^{*}$ quando $d \rightarrow+\infty$.

Prova: Similarmente ao Corolário 1, segue a prova do item (ii), de Montagner, Oliveira, Peres e Bliman (2007, Corolário 2).

Do ponto de vista da factibilidade, os teoremas 2 e 3 fornecem condições convexas de dimensão finita que são suficientes e, para $d$ suficientemente grande, tornam-se também necessárias para a solução dos lemas 2 e 3, respectivamente. Em outras palavras, se os lemas 2 e 3 têm solução, existirá um $d \in \mathbb{N}$ a partir do qual os teoremas 2 e 3 serão factíveis, qualquer que seja a escolha do grau $g \geq 1$.

Em termos da otimalidade, os teoremas 2 e 3 fornecem condições que, com o aumento de $d$, convergem assintoticamente para o mínimo valor do custo garantido sob estabilidade quadrática fornecido pelos lemas 2 e 3, respectivamente.

Assim como no caso de estabilização apenas, a presença de variáveis extras nos teoremas 2 e 3 pode acelerar significativamente a convergência, isto é, para um mesmo $d$, as condições dos teoremas 2 e 3 podem fornecer custos garantidos $\mathscr{H}_{\infty}$ e $\mathscr{H}_{2}$ menores do que as condições sem as variáveis de folga, como por exemplo as condições dadas em Montagner, Oliveira, Peres e Bliman (2007) no contexto de sistemas contínuos variantes no tempo. Além disso, a estrutura polinomial de grau $g$ para o ganho $K(\alpha)$ permite estabilizar quadraticamente o sistema com custos garantidos menores calculados com menor esforço computacional, como ilustrado na próxima seção.

\section{EXPERIMENTOS NUMÉRICOS}

Todos os experimentos foram feitos usando o SeDuMi (Sturm, 1999) e o YALMIP (Löfberg, 2004) com o Matlab versão 7.0.1 em um computador Athlon 64 X2 
$6000+(3.0 \mathrm{GHz}), 2 \mathrm{~GB}$ RAM $(800 \mathrm{MHz})$ com Linux Ubuntu. A implementação numérica de todas as relaxações LMIs apresentadas no artigo está disponível para download em www.dt.fee.unicamp.br/ ricfow/ robust.htm. A complexidade numérica associada aos exemplos é dada em termos do número $V$ de variáveis escalares, o número $L$ de linhas de LMIs, e o tempo computacional (em segundos).

Exemplo 1 Considere o sistema nebuloso de T-S, usado em Fang et al. (2006), definido pelas regras

$$
\begin{array}{ll}
\text { Regra 1: } & \text { SE } x_{1}(t) \text { É } M_{1} \\
& \text { ENTÃO } \dot{x}(t)=A_{1} x(t)+B_{1} u(t) \\
\text { Regra 2: } & \text { SE } x_{1}(t) \text { É } M_{2} \\
& \text { ENTÃO } \dot{x}(t)=A_{2} x(t)+B_{2} u(t) \\
\text { Regra 3: } & \text { SE } x_{1}(t) \dot{E} M_{3} \\
& \text { ENTÂO } \dot{x}(t)=A_{3} x(t)+B_{3} u(t)
\end{array}
$$

sendo

$$
\begin{gathered}
{\left[\begin{array}{c:c:c}
A_{1} & A_{2} & A_{3}
\end{array}\right]=\left[\begin{array}{cc:cc:cc}
1.59 & -7.29 & 0.02 & -4.64 & -a & -4.33 \\
0.01 & 0 & 0.35 & 0.21 & 0 & 0.05
\end{array}\right],} \\
{\left[\begin{array}{l:l:l}
B_{1} & B_{2} & B_{3}
\end{array}\right]=\left[\begin{array}{c:c:c}
1 & 8 & 6-b \\
0 & 0 & -1
\end{array}\right]}
\end{gathered}
$$

Este exemplo, considerado como um benchmark para controle de sistemas nebulosos de T-S, tem sido bastante usado nos artigos recentes que tratam de estabilização de sistemas contínuos. A estratégia adotada aqui é fixar o valor $a=5$ e procurar pelo maior valor de $b$ tal que o sistema seja quadraticamente estabilizável. A condição de estabilização proposta neste trabalho (Teorema 1, denotada por T1) é comparada com a seguintes condições: Kim e Lee (2000) (KL00), Liu e Zhang (2003) (LZZ03), Teixeira et al. (2003) (TAA03), Fang et al. (2006) (FLKHL06), Montagner, Oliveira, Peres e Bliman (2007) (MOPB07), Montagner, Oliveira e Peres (2007) (MOP07), Sala e Ariño (2007) (SA07) e Montagner et al. (2009) (MOP09). A Tabela 1 mostra os máximos valores de $b$ obtidos e as respectivas complexidades computacionais associadas. Como pode ser visto, a condição do Teorema 1 com $g=3$ estabiliza quadraticamente o sistema com o maior valor possível de $b$ e o menor esforço computacional quando comparada com as condições de SA07 e MOPB07 (que também chegaram no mesmo valor ou muito próximo). Vale a pena ressaltar que o controlador nebuloso estabilizante obtido pela condição do Teorema 1 é polinomial de grau três enquanto que o controlador obtido em SA07 é de grau um (ou seja, um compensador paralelo distribuído convencional).
Tabela 1: Máximos valores de $b$ e complexidades computacionais obtidas no problema de estabilização dado no Exemplo 1. $V$ é o número de variáveis escalares e $L$ é o número de linhas de LMls. O tempo computacional é dado em segundos.

\begin{tabular}{c|c|c|c|c}
\hline \hline Método & $b_{\max }$ & $V$ & $L$ & Tempo (s) \\
\hline KL00 & 0.322 & 27 & 20 & 0.06 \\
LZ03 & 3.085 & 33 & 20 & 0.05 \\
MOP07 $_{d=5}$ & 3.219 & 9 & 326 & 0.08 \\
TAA03 & 6.777 & 63 & 38 & 0.12 \\
FLKHL06 & 8.114 & 72 & 40 & 0.07 \\
MOP09 $_{g=d=5}$ & 8.347 & 177 & 314 & 0.60 \\
MOPB07 $_{g=d=5}$ & 8.489 & 45 & 158 & 0.19 \\
SA07 & 8.490 & 504 & 86 & 0.44 \\
T1 $1_{g=3, d=0}$ & 8.490 & 103 & 62 & 0.11 \\
\hline \hline
\end{tabular}

Exemplo 2 Considere os subsistemas de um sistema nebuloso de T-S dados por

$$
\begin{aligned}
& {\left[\begin{array}{l:l}
A_{1} & A_{2}
\end{array}\right]=\left[\begin{array}{lll:lll}
0.32 & 0.60 & 0.51 & 0.68 & 0.41 & 0.96 \\
0.99 & 0.62 & 0.81 & 0.18 & 0.32 & 0.15 \\
1.00 & 0.43 & 0.03 & 0.95 & 0.29 & 0.29
\end{array}\right]} \\
& {\left[\begin{array}{l:l}
B_{1} & B_{2}
\end{array}\right]=\left[\begin{array}{l:l}
0.68 & 0.57 \\
0.76 & 0.44 \\
0.38 & 0.51
\end{array}\right],\left[E_{1}: E_{2}\right]=\left[\begin{array}{c:c}
1 & 0.1 \\
0 & 0 \\
0 & 0
\end{array}\right]} \\
& C_{1}=C_{2}=\left[\begin{array}{lll}
1 & 0 & 0
\end{array}\right],\left[D_{1}: D_{2}\right]=[0.94: 0.14]
\end{aligned}
$$

Esses subsistemas foram gerados aleatoriamente com o objetivo de mostrar a vantagem de usarem-se variáveis de folga em projeto de controladores nebulosos envolvendo a otimização de um critério de desempenho. Neste caso, é usada a norma $\mathscr{H}_{2}$ como critério e as relaxações do Teorema 3 (T3) são comparadas com as relaxações de Montagner, Oliveira, Peres e Bliman (2007, Teorema 4, eq. (43)-(45)) (MOPB07), que não utilizam variáveis de folga. A Figura 1 mostra os resultados considerando $g=1, \ldots, 3$ para MOPB07 e somente $g=1$ para T3. Para cada grau, são aplicadas as relaxações de Pólya para $d=1, \ldots, 10$.

Como pode ser visto, usando $g=1$ no T3 é possível obter um custo garantido ótimo $\mathscr{H}_{2}$ (o mesmo valor é obtido para graus maiores). Por outro lado, os graus $g=1, \ldots, 3$ e dez relaxações de Pólya nas condições de MOPB07 não foram suficientes para chegar ao mesmo resultado. Isto mostra como o uso de variáveis de folga pode acelerar significativamente a convergência das relaxações. Para fins ilustrativos, a Figura 2 mostra uma simulação no tempo do sistema controlado pelo ganhos

$$
\begin{aligned}
& K_{10}=\left[\begin{array}{lll}
-3.6637 & -0.4753 & -4.1635
\end{array}\right] \\
& K_{01}=\left[\begin{array}{lll}
-1.9687 & -0.8109 & -1.4531
\end{array}\right]
\end{aligned}
$$




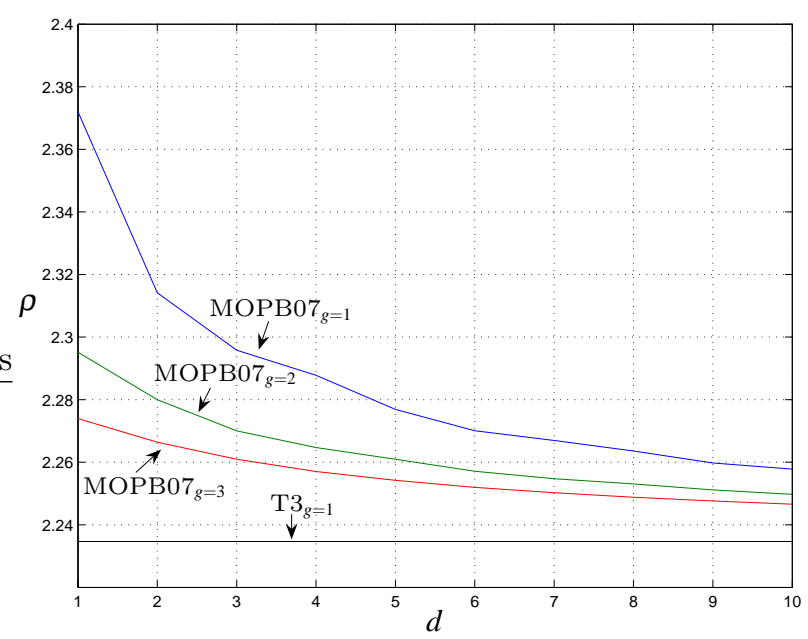

Figura 1: Custos garantidos $\mathscr{H}_{2}$ obtidos pelas condições do T3 e pelas condições de MOPB07 para o Exemplo 2.

que foram obtidos pelo T3 para $g=1, d=0$. O valor do custo garantido $\mathscr{H}_{2}$ é $\rho=2.2347$. Para a simulação, foram consideradas as funções

$$
\begin{gathered}
\alpha_{1}(t)=0.5 \cos \left(10 x_{1}(t)\right)+0.5, \\
\alpha_{2}(t)=-0.5 \cos \left(10 x_{1}(t)\right)+0.5
\end{gathered}
$$

com a condição inicial $x_{0}=\left[\begin{array}{lll}1 & -1 & -0.5\end{array}\right]^{\prime}$.
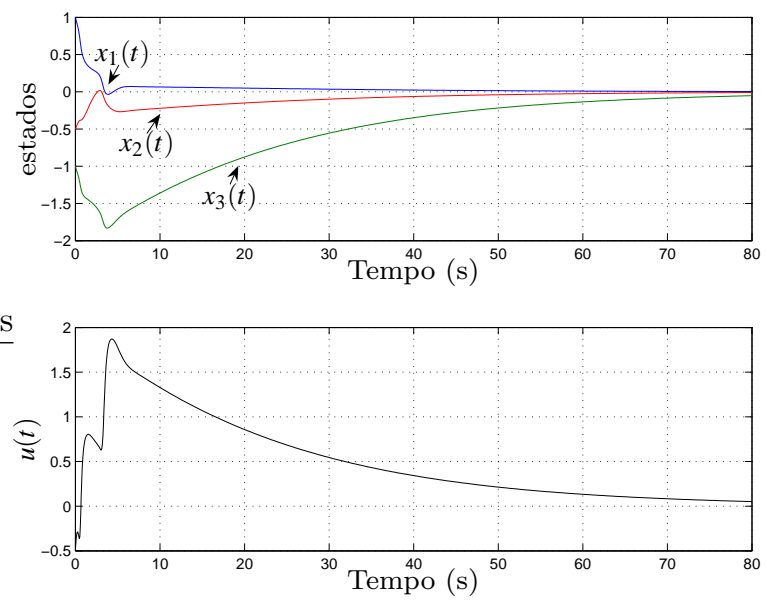

Figura 2: Simulação no tempo do sistema do Exemplo 2 com os ganhos dados em (32).

\section{CONCLUSÃO}

Neste trabalho foram propostas condições na forma de desigualdades matriciais lineares (LMIs) que permitem a determinação de controladores de realimentação de estados quadraticamente estabilizantes para sistemas nebulosos de T-S contínuos no tempo. As LMIs são construídas com variáveis extras, oriundas de uma formulação para a condição de estabilizabilidade baseada no Lema de Finsler, em termos do grau de relaxação $d$ (ligado ao Teorema de Pólya) e do grau $g$ do controlador polinomial a ser obtido. Um controlador quadraticamente estabilizante é obtido para um nível de relaxação $d$ suficientemente grande sempre que tal controlador existir. Extensões para controle com custo ótimo $\mathscr{H}_{2}$ e $\mathscr{H}_{\infty}$ são também apresentadas. Comparações numéricas com resultados da literatura ilustram a superioridade da abordagem proposta.

A metodologia apresentada pode ser estendida para tratar síntese de controladores para sistemas nebulosos de T-S com taxas de variações limitadas das funções de pertinência, por meio de funções de Lyapunov quadráticas no estado e polinomiais nas variáveis de premissa.

\section{AGRADECIMENTOS}

Às agências FAPESP, CAPES e CNPq pelo apoio financeiro.

\section{REFERÊNCIAS}

Andrea, C. Q., Assunção, E., Pinto, J. O. P., Teixeira, M. C. M. e Junior, L. G. (2008). Controle ótimo $\mathscr{H}_{\infty}$ de sistemas não-lineares com modelos fuzzy TakagiSugeno, SBA: Controle \& Automação 19(3): 256269.

Apkarian, P. e Gahinet, P. (1995). A convex characterization of gain-scheduled $\mathscr{H}_{\infty}$ controllers, IEEE Transactions on Automatic Control 40(5): 853864 .

Apkarian, P., Gahinet, P. e Becker, G. (1995). Self-scheduled $\mathscr{H}_{\infty}$ control of linear parametervarying systems: a design example, Automatica 31(9): 1251-1261.

Arrifano, N. S. D., Oliveira, V. A. e Cossi, L. V. (2006). Synthesis of an LMI-based fuzzy control system with guaranteed cost performance: a piecewise Lyapunov approach, SBA: Controle \& $A u$ tomação 17(2): 213-225.

Avellar, G. S. C., Mozelli, L. A. e Palhares, R. M. (2008). Condições LMIs alternativas para sistemas TakagiSugeno via função de Lyapunov fuzzy, Anais do XVI Congresso Brasileiro de Automática, Juiz de Fora, MG, Brasil.

Bernussou, J., Peres, P. L. D. e Geromel, J. C. (1989). A linear programming oriented procedure for quadratic stabilization of uncertain systems, Systems \& Control Letters 13(1): 65-72. 
Bliman, P.-A., Oliveira, R. C. L. F., Montagner, V. F. e Peres, P. L. D. (2006). Existence of homogeneous polynomial solutions for parameter-dependent linear matrix inequalities with parameters in the simplex, Proceedings of the 45th IEEE Conference on Decision and Control, San Diego, CA, USA, pp. 1486-1491.

Boyd, S., El Ghaoui, L., Feron, E. e Balakrishnan, V. (1994). Linear Matrix Inequalities in System and Control Theory, SIAM Studies in Applied Mathematics, Philadelphia, PA.

Cao, Y. e Frank, P. M. (2000). Analysis and synthesis of nonlinear time-delay systems via fuzzy control approach, IEEE Transactions on Fuzzy Systems 8(2): 200-211.

Chen, B., Tseng, C. e Uang, H. (2000). Mixed $\mathscr{H}_{2} / \mathscr{H}_{\infty}$ fuzzy output feedback control design for nonlinear dynamic systems: an LMI approach, IEEE Transactions on Fuzzy Systems 8(3): 249-265.

de Oliveira, M. C. e Skelton, R. E. (2001). Stability tests for constrained linear systems, in $\mathrm{S}$. O. Reza Moheimani (ed.), Perspectives in Robust Control, Vol. 268 of Lecture Notes in Control and Information Science, Springer-Verlag, New York, pp. 241257.

de Souza, C. E., Trofino, A. e de Oliveira, J. (2003). Parametric Lyapunov function approach to $\mathscr{H}_{2}$ analysis and control of linear parameter-dependent systems, IEE Proceedings - Control Theory and Applications 150(5): 501-508.

Fang, C.-H., Liu, Y.-S., Kau, S.-W., Hong, L. e Lee, C.-H. (2006). A new LMI-based approach to relaxed quadratic stabilization of T-S fuzzy control systems, IEEE Transactions on Fuzzy Systems 14(3): 386-397.

Feng, G. (2006). A survey on analysis and design of model-based fuzzy control systems, IEEE Transactions on Fuzzy Systems 14(5): 676-697.

Green, M. e Limebeer, D. J. N. (1995). Linear Robust Control, Prentice-Hall, Englewood Cliffs, NJ.

Hardy, G. H., Littlewood, J. E. e Pólya, G. (1952). Inequalities, 2 edn, Cambridge University Press, Cambridge, UK.

Johansson, M., Rantzer, A. e Arzen, K. E. (1999). Piecewise quadratic stability of fuzzy systems, IEEE Transactions on Fuzzy Systems 7(6): 713-722.
Kau, S.-W., Liu, Y.-S., Hong, L., Lee, C.-H., Fang, C.H. e Lee, L. (2005). A new LMI condition for robust stability of discrete-time uncertain systems, Systems \& Control Letters 54(12): 1195-1203.

Kim, E. e Lee, H. (2000). New approaches to relaxed quadratic stability condition of fuzzy control systems, IEEE Transactions on Fuzzy Systems 8(5): 523-534.

Kumar, P. P., Kar, I. e Behera, L. (2006). Variablegain controllers for nonlinear systems using the T-S fuzzy model, IEEE Transactions on Systems, Man, and Cybernetics, Part B: Cybernetics 36(6): 14421449.

Lam, H. K. e Leung, F. H. F. (2007). LMI-based stability and performance conditions for continuoustime nonlinear systems in Takagi-Sugeno's form, IEEE Transactions on Systems, Man, and Cybernetics, Part B: Cybernetics 37(5): 1396-1406.

Lian, K., Chiang, T., Chiu, C. e Liu, P. (2001). Synthesis of fuzzy model-based designs to synchronization and secure communications for chaotic systems, IEEE Transactions on Systems, Man, and Cybernetics, Part B: Cybernetics 31(1): 66-83.

Lin, C., Wang, Q.-G. e Lee, T. H. (2006). Less conservative $\mathscr{D}$-stability test for polytopic systems using linearly parameter-dependent Lyapunov functions, IEE Proceedings - Control Theory and Applications 153(6): 665-670.

Liu, X. e Zhang, Q. (2003). New approaches to $\mathscr{H}_{\infty}$ controller designs based on fuzzy observers for T-S fuzzy systems via LMI, Automatica 39(5): 15711582 .

Löfberg, J. (2004). YALMIP: A toolbox for modeling and optimization in MATLAB, Proceedings of the 2004 IEEE International Symposium on Computer Aided Control Systems Design, Taipei, Taiwan, pp. 284-289. http://control.ee.ethz. ch/ joloef/yalmip.php.

Montagner, V. F., Oliveira, R. C. L. F. e Peres, P. L. D. (2007). Necessary and sufficient LMI conditions to compute quadratically stabilizing state feedback controllers for Takagi-Sugeno systems, Proceedings of the 2007 American Control Conference, New York, NY, USA, pp. 4059-4064.

Montagner, V. F., Oliveira, R. C. L. F. e Peres, P. L. D. (2008). Relaxações LMI para o projeto convexo de controladores $\mathscr{H}_{\infty}$ para sistemas nebulosos de Takagi-Sugeno, Anais do XVI Congresso Brasileiro de Automática, Juiz de Fora, MG, Brasil. 
Montagner, V. F., Oliveira, R. C. L. F. e Peres, P. L. D. (2009). Convergent LMI relaxations for quadratic stabilizability and $\mathscr{H}_{\infty}$ control of Takagi-Sugeno fuzzy systems, IEEE Transactions on Fuzzy Systems . to appear.

Montagner, V. F., Oliveira, R. C. L. F., Peres, P. L. D. e Bliman, P.-A. (2007). Linear matrix inequality characterisation for $\mathscr{H}_{\infty}$ and $\mathscr{H}_{2}$ guaranteed cost gainscheduling quadratic stabilisation of linear timevarying polytopic systems, IET Control Theory 86 Applications 1(6): 1726-1735.

Montagner, V. F. e Peres, P. L. D. (2006). State feedback gain scheduling for linear systems with time-varying parameters, Journal of Dynamic Systems, Measurement and Control - Transactions of ASME 128(2): 365-370.

Mozelli, L. A., Palhares, R. M. e Avellar, G. S. C. (2008). Novas condições de estabilidade e de estabilização para sistemas Takagi-Sugeno baseadas na função de Lyapunov fuzzy, Anais do XVI Congresso Brasileiro de Automática, Juiz de Fora, MG, Brasil.

Mozelli, L. A., Palhares, R. M. e Avellar, G. S. C. (2009). A systematic approach to improve multiple Lyapunov function stability and stabilization conditions for fuzzy systems, Information Sciences 179(8): 1149-1162.

Mozelli, L. A., Palhares, R. M., Souza, F. O. e Mendes, E. M. A. M. (2009). Reducing conservativeness in recent stability conditions of TS fuzzy systems, $A u$ tomatica 45(6): 1580-1583.

Nguang, S. K. e Shi, P. (2003). $\mathscr{H}_{\infty}$ fuzzy output feedback control design for nonlinear systems: an LMI approach, IEEE Transactions on Fuzzy Systems 11(3): 331-340.

Oliveira, R. C. L. F. e Peres, P. L. D. (2005). Stability of polytopes of matrices via affine parameterdependent Lyapunov functions: Asymptotically exact LMI conditions, Linear Algebra and Its Applications 405: 209-228.

Oliveira, R. C. L. F. e Peres, P. L. D. (2007). Parameterdependent LMIs in robust analysis: characterization of homogeneous polynomially parameterdependent solutions via LMI relaxations, IEEE Transactions on Automatic Control 52(7): 13341340 .

Powers, V. e Reznick, B. (2001). A new bound for Pólya's Theorem with applications to polynomials positive on polyhedra, Journal of Pure and Applied Algebra 164: 221-229.
Prajna, S., Papachristodoulou, A., Seiler, P. e Parrilo, P. A. (2004). SOSTOOLS: Sum of squares optimization toolbox for MATLAB.

Rhee, B.-J. e Won, S. (2006). A new fuzzy Lyapunov function approach for a Takagi-Sugeno fuzzy control system design, Fuzzy Sets and Systems 157(9): 1211-1228.

Sala, A. e Ariño, C. (2007). Asymptotically necessary and sufficient conditions for stability and performance in fuzzy control: Applications of Polya's theorem, Fuzzy Sets and Systems 158(24): 2671-2686.

Scherer, C. W. (2001). LPV control and full block multipliers, Automatica 37(3): 361-375.

Scherer, C. W. (2003). Higher-order relaxations for robust LMI problems with verifications for exactness, Proceedings of the 42nd IEEE Conference on Decision and Control, Maui, HI, USA, pp. 4652-4657.

Scherer, C. W. (2005). Relaxations for robust linear matrix inequality problems with verifications for exactness, SIAM Journal on Matrix Analysis and Applications 27(2): 365-395.

Scherer, C. W. e Hol, C. W. J. (2006). Matrix sum-of-squares relaxations for robust semi-definite programs, Mathematical Programming: Series B 107(1-2): 189-211.

Sturm, J. F. (1999). Using SeDuMi 1.02, a MATLAB toolbox for optimization over symmetric cones, $O p$ timization Methods and Software 11-12: 625-653. http://sedumi.mcmaster.ca/.

Tanaka, K., Ikeda, T. e Wang, H. O. (1998a). Fuzzy regulators and fuzzy observers: relaxed stability conditions and LMI-based designs, IEEE Transactions on Fuzzy Systems 6(2): 250-265.

Tanaka, K., Ikeda, T. e Wang, H. O. (1998b). A unified approach to controlling chaos via an LMI-based fuzzy control system design, IEEE Transactions on Circuits and Systems Part I: Fundamental Theory and Applications 45(10): 1021-1040.

Tanaka, K., Ohtake, H. e Wang, H. O. (2007). A descriptor system approach to fuzzy control system design via fuzzy Lyapunov functions, IEEE Transactions on Fuzzy Systems 15(3): 333-341.

Tanaka, K., Ohtake, H. e Wang, H. O. (2009). Guaranteed cost control of polynomial fuzzy systems via a sum of squares approach, IEEE Transactions on Systems, Man, and Cybernetics, Part B: Cybernetics 39(2): 561-567. 
Tanaka, K. e Wang, H. (2001). Fuzzy Control Systems Design and Analysis: A Linear Matrix Inequality Approach, John Wiley \& Sons, Inc., New York.

Teixeira, M. C. M., Assunção, E. e Avellar, R. G. (2003). On relaxed LMI-based designs for fuzzy regulators and fuzzy observers, IEEE Transactions on Fuzzy Systems 11(5): 613-623.

Teixeira, M. C. M., Pietrobom, H. C. e Assunção, E. (2000). Novos resultados sobre a estabilidade e controle de sistemas não-lineares utilizando modelos fuzzy e LMI, SBA: Controle \&3 Automação 11(2): $37-48$.

Teixeira, M. C. M. e Zak, S. H. (1999). Stabilizing controller design for uncertain nonlinear systems using fuzzy models, IEEE Transactions on Fuzzy Systems 7(2): 133-142.

Tuan, H. D., Apkarian, P., Narikiyo, T. e Yamamoto, Y. (2001). Parameterized linear matrix inequality techniques in fuzzy control system design, IEEE Transactions on Fuzzy Systems 9(2): 324-332.

Wang, F. e Balakrishnan, V. (2002). Improved stability analysis and gain-scheduled controller synthesis for parameter-dependent systems, IEEE Transactions on Automatic Control 47(5): 720-734.

Wang, H. O., Tanaka, K. e Griffin, M. F. (1996). An approach to fuzzy control of nonlinear systems: stability and design issues, IEEE Transactions on Fuzzy Systems 4(1): 14-23.

Xu, S. e Lam, J. (2005). Robust $\mathscr{H}_{\infty}$ control for uncertain discrete-time-delay fuzzy systems via output feedback controllers, IEEE Transactions on Fuzzy Systems 13(2): 82-93.

Yang, G.-H. e Dong, J. (2008). Robust stability of polytopic systems via affine parameter-dependent Lyapunov functions, SIAM Journal on Control and Optimization 47(5): 2642-2662. 